Article

\title{
Some General Theorems for Compact Acyclic Multifunctions
}

\section{Donal O'Regan}

School of Mathematics, Statistics and Applied Mathematics, National University of Ireland, H91 TK33 Galway, Ireland; donal.oreganl@nuigalway.ie

Received: 6 July 2019; Accepted: 30 July 2019; Published: 31 July 2019

Abstract: We present general Leray-Schauder type theorems for compact acyclic Multifunctions, using the topological transversality theorem by the author.

Keywords: essential maps; acyclic maps; fixed points

MSC: 47H10; 54H25

\section{Introduction}

If $F$ and $G$ are continuous compact single valued maps and $F \cong G$, then $F$ is essential [1] if and only if $G$ is essential. This result was extended to multimaps in a variety of settings; see [2,3] and the references therein. In this paper, using the topological transversality by the author for compact acyclic maps [2,4-7], we establish a variety of Leray-Schauder type theorems which are useful from an application viewpoint. Please note that essential maps automatically generate fixed point results for these maps. As a result, our theory can be applied when considering variation methods, iteration methods, perturbation methods, degree theory methods, upper and lower solution methods, etc. (see for example [8-10]). Many problems which arise naturally in applications can be formulated in the form $x \in F x$ and we can relate it to a simpler problem via the family of problems $x \in \lambda F x$, $0 \leq \lambda \leq 1$. If the zero map is essential then under appropriate conditions (see our Leray-Schauder type alternatives) $F$ will be essential (so it automatically has a fixed point).

Let $H$ be the Čech homology functor with compact carriers and coefficients in the field of rational numbers $K$ from the category of Hausdorff topological spaces and continuous maps to the category of graded vector spaces and linear maps of degree zero. Now $H(X)=\left\{H_{q}(X)\right\}$ (where $X$ is a Hausdorff topological space) is a graded vector space, $H_{q}(X)$ being the $q$-dimensional Čech homology group with compact carriers of $X$. For a continuous map $f: X \rightarrow X, H(f)$ is the induced linear map $f_{\star}=\left\{f_{\star q}\right\}$ where $f_{\star q}: H_{q}(X) \rightarrow H_{q}(X)$. A space $X$ is called acyclic if $X$ is nonempty, $H_{q}(X)=0$ for every $q \geq 1$, and $H_{0}(X) \approx K$. Let $X$ and $Z$ be subsets of Hausdorff topological spaces. Consider a map $F: X \rightarrow K(Z)$ where $K(Z)$ denotes the family of nonempty compact subsets of $Z$. Now $F: X \rightarrow K(Z)$ is called acyclic if $F$ is upper semicontinuous (u.s.c.) with acyclic values.

\section{Topological Transversality Theorem}

We begin this section with essential maps. Let $E$ be a completely regular topological space (i.e., a Tychonoff space) and $U$ an open subset of $E$ (now $\bar{U}$ is the closure of $U$ in $E$ and $\partial U$ the boundary of $U$ in $E$ ).

Definition 1. Write $F \in A C(\bar{U}, E)$ if $F: \bar{U} \rightarrow K(E)$ is an acyclic compact map.

Definition 2. Write $F \in A C_{\partial U}(\bar{U}, E)$ if $F \in A C(\bar{U}, E)$ with $x \notin F(x)$ when $x \in \partial U$. 
Definition 3. Considering two maps $F, G \in A C_{\partial U}(\bar{U}, E)$ and they are said to be homotopic in $A C_{\partial U}(\bar{U}, E)$, written $F \cong G$ in $A C_{\partial U}(\bar{U}, E)$ if there exists a u.s.c. compact map $\Psi: \bar{U} \times[0,1] \rightarrow K(E)$ with $\Psi_{t} \in$ $A C_{\partial U}(\bar{U}, E)$ for each $t \in[0,1], \Psi_{1}=F$ and $\Psi_{0}=G$ (where $\Psi_{t}(x)=\Psi(t, x)$ ).

Note $\cong$ is an equivalence relation in $A C_{\partial U}(\bar{U}, E)$.

Definition 4. A map $F \in A C_{\partial U}(\bar{U}, E)$ is essential in $A C_{\partial U}(\bar{U}, E)$ if for any $G \in A C_{\partial U}(\bar{U}, E)$ with $\left.G\right|_{\partial U}=\left.F\right|_{\partial U}$ and with $G \cong F$ in $A C_{\partial U}(\bar{U}, E)$ there exists a $x \in U$ with $x \in G(x)$.

In [4] we established the topological transversality theorem.

Theorem 1. Consider two maps $F$ and $G$ in $A C_{\partial U}(\bar{U}, E)$ with $F \cong G$ in $A C_{\partial U}(\bar{U}, E)$. Now $F$ is essential in $A C_{\partial U}(\bar{U}, E)$ iff $G$ is essential in $A C_{\partial U}(\bar{U}, E)$.

We now present two ways of proceeding from here.

\section{Approach 1.}

This approach is motivated from $[4,7]$ (here we present a more general result). We consider the question: If $F$ and $G$ are two maps in $A C_{\partial U}(\bar{U}, E)$ with $\left.G\right|_{\partial U}=\left.F\right|_{\partial U}$ is $F \cong G$ in $A C_{\partial U}(\bar{U}, E)$ ? We will now show that this is true if $E$ is a topological (Hausdorff) vector space, $U$ is convex and

$$
\text { there exists a retraction (continuous) } r: \bar{U} \rightarrow \partial U \text {. }
$$

[Note if $E$ is an infinite dimensional Banach space and $U$ is convex then [7] we know (1) holds].

Let $F, G$ be two maps in $A C_{\partial U}(\bar{U}, E)$ with $\left.G\right|_{\partial U}=\left.F\right|_{\partial U}$ and let $r$ be as in (1). Consider $F^{\star}$ defined by $F^{\star}(x)=F(r(x)), x \in \bar{U}$. Note $F^{\star}(x)=G(r(x)), x \in \bar{U}$ since $\left.G\right|_{\partial U}=\left.F\right|_{\partial u}$. With

$$
\Lambda(x, \lambda)=G(2 \lambda r(x)+(1-2 \lambda) x)=G \circ j(x, \lambda) \text { for }(x, \lambda) \in \bar{U} \times\left[0, \frac{1}{2}\right]
$$

(here $j: \bar{U} \times\left[0, \frac{1}{2}\right] \rightarrow \bar{U}$ (note $\bar{U}$ is convex) is $j(x, \lambda)=2 \lambda r(x)+(1-2 \lambda) x$ ) it is immediate that

$$
G \cong F^{\star} \text { in } A C_{\partial U}(\bar{U}, E)
$$

note $\Lambda: \bar{U} \times\left[0, \frac{1}{2}\right] \rightarrow K(E)$ is a u.s.c. compact map and for any fixed $x \in \bar{U}$ and $t \in\left[0, \frac{1}{2}\right]$ note $\Lambda_{t}(x)=G(j(x, t))$ has acyclic values and finally note if $x \in \partial U$ and $\lambda \in\left[0, \frac{1}{2}\right]$ with $x \in \Lambda_{\lambda}(x)$ then $x \in G(2 \lambda x+(1-2 \lambda) x)=G(x)$, a contradiction.

With

$$
\Theta(x, \lambda)=F((2-2 \lambda) r(x)+(2 \lambda-1) x) \text { for }(x, \lambda) \in \bar{U} \times\left[\frac{1}{2}, 1\right]
$$

we have

$$
F^{\star} \cong F \text { in } A C_{\partial U}(\bar{U}, E) .
$$

Consequently $G \cong F$ in $A C_{\partial U}(\bar{U}, E)$.

In this situation we replace Definition 4 with:

Definition 5. A map $F \in A C_{\partial U}(\bar{U}, E)$ is essential in $A C_{\partial U}(\bar{U}, E)$ if any map $G \in A C_{\partial U}(\bar{U}, E)$ with $\left.G\right|_{\partial U}=\left.F\right|_{\partial U}$ there exists a $x \in U$ with $x \in G(x)$.

Recall topological vector spaces are Tychonoff so from Theorem 1 we have:

Theorem 2. Let $E$ be a topological vector space, $U$ an open convex subset of $E$ and suppose (1) holds. Suppose $F, G \in A C_{\partial U}(\bar{U}, E)$ with $F \cong G$ in $A C_{\partial U}(\bar{U}, E)$. Now $F$ is essential (Definition 5) in $A C_{\partial U}(\bar{U}, E)$ iff $G$ is essential (Definition 5) in $A C_{\partial U}(\bar{U}, E)$.

From Theorem 2 we present very general Leray-Schauder type results. 
Definition 6. Write $F \in A C(E, E)$ if $F: E \rightarrow K(E)$ is an acyclic compact map.

Theorem 3. Let $E$ be a topological vector space, $U$ an open convex subset of $E,(1)$ holds and $F \in A C_{\partial U}(\bar{U}, E)$. Assume $J \in A C(E, E)$ with

$$
z \notin J(z) \text { for } z \in E \backslash U
$$

and

$$
\text { for any map } \Phi \in A C(E, E) \text { there exists } y \in E \text { with } y \in \Phi(y) \text {. }
$$

Suppose $F \cong J$ in $A C_{\partial U}(\bar{U}, E)$. Then $F$ is essential (Definition 5) in $A C_{\partial U}(\bar{U}, E)$.

Proof. We show $J$ is essential (Definition 5) in $A C_{\partial U}(\bar{U}, E)$. If this is true then $F$ is essential (Definition 5) in $A C_{\partial U}(\bar{U}, E)$ from Theorem 2. Let $G \in A C_{\partial U}(\bar{U}, E)$ with $\left.G\right|_{\partial U}=\left.J\right|_{\partial U}$. We need to show that $G$ has a fixed point in $U$. Let

$$
\Phi(x)=\left\{\begin{array}{l}
G(x), x \in \bar{U} \\
J(x), x \in E \backslash \bar{U} .
\end{array}\right.
$$

Note $\Phi \in A C(E, E)$ so from (3) we have a $z \in E$ with $z \in \Phi(z)$. Now (2) yields $z \in U$ so $z \in G(z)$.

Corollary 1. Let $E$ be a topological vector space, $U$ an open convex subset of $E$, (1) holds, $F \in A C_{\partial U}(\bar{U}, E)$, $u_{0} \in U$ and suppose (3) holds. Suppose $F \cong\left\{u_{0}\right\}$ in $A C_{\partial U}(\bar{U}, E)$. Then $F$ is essential (Definition 5) in $A C_{\partial U}(\bar{U}, E)$.

Proof. Let $J(x)=\left\{u_{0}\right\}$ for $x \in E$ and the result follows from Theorem 3 .

Remark 1. (i). For spaces E which satisfy (3) we refer the reader to [11].

(ii). Without loss of generality, take $u_{0}=0$. Note if $E$ is a completely metrizable locally convex space and $x \notin t F(x)$ for $x \in \partial U$ and $t \in(0,1)$ then one homotopy from $F$ to $\{0\}$ is $\Psi(x, t)=t F(x)$ (here $t \in[0,1]$ and $x \in \bar{U}$ ). To see this note $\Psi: \bar{U} \times[0,1] \rightarrow K(E)$ is a u.s.c. compact (see [12], Theorem 4.18) map and also note for a fixed $t \in[0,1]$ and a fixed $x \in \bar{U}$ that $\Psi_{t}(x)$ is acyclic valued (recall homeomorphic spaces have isomorphic homology groups) so $\Psi_{t} \in A C_{\partial U}(\bar{U}, E)$. [Note E being a completely metrizable locally convex space can be replaced by any (Hausdorff) topological vector space E which has the property that the closed convex hull of a compact set in $E$ is compact].

\section{Approach 2.}

Here we do not assume $E$ is a topological vector space and we do not assume (1). In this approach, instead of concentrating on homotopies, we will consider essential maps and spaces in general.

From Theorem 1, we present very general Leray-Schauder type results.

Definition 7. Consider two maps $F, J \in A C(E, E)$ and they are said to be homotopic in $A C(E, E)$, written $F \cong J$ in $A C(E, E)$, if there exists a u.s.c. compact map $R: E \times[0,1] \rightarrow K(E)$ with $R_{t} \in A C(E, E)$ for each $t \in[0,1], R_{1}=F$ and $R_{0}=J$ (where $R_{t}(x)=R(x, t)$ ).

Theorem 4. Let $E$ be a completely regular topological space, $U$ an open subset of $E$ and suppose $F \in$ $A C_{\partial U}(\bar{U}, E)$. Assume $J \in A C(E, E)$ with (2) holding and suppose

$$
\text { there exists } y \in U \text { with } y \in J(y)
$$

and 
for any $\Phi \in A C(E, E)$ with $\Phi \cong J$ in $A C(E, E)$

there exists $z \in E$ with $z \in \Phi(z)$.

Suppose $F \cong J$ in $A C_{\partial U}(\bar{U}, E)$. Then $F$ is essential (Definition 4) in $A C_{\partial U}(\bar{U}, E)$.

Proof. We show $J$ is essential (Definition 4) in $A C_{\partial U}(\bar{U}, E)$ (so then $F$ is essential (Definition 4) in $A C_{\partial U}(\bar{U}, E)$ from Theorem 1). Let $G \in A C_{\partial U}(\bar{U}, E)$ with $\left.G\right|_{\partial U}=\left.J\right|_{\partial U}$ and with $G \cong J$ in $A C_{\partial U}(\bar{U}, E)$. We need to show $G$ has a fixed point in $U$. There exists (see Definition 3 ) a u.s.c. compact map $\Theta: \bar{U} \times[0,1] \rightarrow K(E)$ with $\Theta_{t} \in A C_{\partial U}(\bar{U}, E)$ for each $t \in[0,1], \Theta_{0}=J$ and $\Theta_{1}=G$ and let

$$
\Omega=\left\{x \in \bar{U}: x \in \Theta_{t}(x) \text { for some } t \in[0,1]\right\} .
$$

Notice $\Omega \neq \varnothing$ (see (4)) is closed and compact and $\Omega \cap(E \backslash U)=\varnothing$ (note $\Theta_{t} \in A C_{\partial U}(\bar{U}, E)$ for $t \in[0,1])$. Thus, there exists a continuous map $\sigma: E \rightarrow[0,1]$ with $\sigma(\Omega)=1$ and $\sigma(E \backslash U)=0$. Define $\Psi: E \times[0,1] \rightarrow K(E)$ by

$$
\Psi(x, t)=\left\{\begin{array}{l}
\Theta(x, t \sigma(x)), \quad x \in \bar{U} \\
J(x), \quad x \in E \backslash U .
\end{array}\right.
$$

Note $\Psi: E \times[0,1] \rightarrow K(E)$ is an upper semicontinuous compact map with $\Psi_{t} \in A C(E, E)$ for each $t \in[0,1]$, so as a result $\Psi_{1} \cong \Psi_{0}=J$ in $A C(E, E)$. From (5) we have a $x \in E$ with $x \in \Psi_{1}(x)$. If $x \in E \backslash \bar{U}$ then $x \in J(x)$ which contradicts (2). Consequently $x \in U$ so $x \in \Lambda(x, \sigma(x))$ and as a result $x \in \Omega$ which implies $\sigma(x)=1$ and so $x \in \Lambda(x, 1)=G(x)$.

Corollary 2. Let $E$ be a completely regular topological space, $U$ an open subset of $E, u_{0} \in U$ and suppose $F \in A C_{\partial U}(\bar{U}, E)$. Assume

$$
\begin{aligned}
& \text { for any } \Phi \in A C(E, E) \text { with } \Phi \cong\left\{u_{0}\right\} \text { in } A C(E, E) \\
& \text { there exists } z \in E \text { with } z \in \Phi(z) \text {. }
\end{aligned}
$$

Suppose $F \cong\left\{u_{0}\right\}$ in $A C_{\partial U}(\bar{U}, E)$. Then $F$ is essential (Definition 4) in $A C_{\partial U}(\bar{U}, E)$.

Proof. Let $J(x)=\left\{u_{0}\right\}$ for $x \in E$ and apply Theorem 4 .

Of course if (3) holds then automatically (6) holds. We now give a result where $\Phi \cong\left\{u_{0}\right\}$ in $A C(E, E)$ plays a major role.

Theorem 5. Let $E$ be a (metrizable) $A N R, U$ an open subset of $E, u_{0} \in U, F \in A C_{\partial U}(\bar{U}, E)$ and suppose $F \cong\left\{u_{0}\right\}$ in $A C_{\partial U}(\bar{U}, E)$. Then $F$ is essential (Definition 4) in $A C_{\partial U}(\bar{U}, E)$.

Proof. It follows immediately from Corollary 2. once we show (6). Let $\Phi \in A C(E, E)$ with $\Phi \cong\left\{u_{0}\right\}$ in $A C(E, E)$, so (see Definition 7) there exists a u.s.c. compact map $R: E \times[0,1] \rightarrow K(E)$ with $R_{t} \in A C(E, E)$ for each $t \in[0,1], R_{1}=\Phi$ and $R_{0}=\left\{u_{0}\right\}$. Note $E$ can be regarded as a closed subset of a normed space $X$ (see the Arens-Eells theorem). Since $E \in A N R$ there is an open neighborhood $V$ of $E$ in $X$ and a retraction (continuous) $r: \bar{V} \rightarrow E$. Let $\lambda: X \rightarrow[0,1]$ be a (continuous) function with $\lambda(X \backslash V)=0$ and $\lambda(E)=1$ and let

$$
Q(x)=\left\{\begin{array}{l}
R(r(x), \lambda(x)), x \in \bar{V} \\
\left\{u_{0}\right\}, x \in X \backslash V
\end{array}\right.
$$

(note if $x \in \partial V$ then $Q(x)=R(r(x), \lambda(x))=R(r(x), 0)=R_{0}(r(x))=\left\{u_{0}\right\}$ ). For fixed $x \in X$ note $Q(x)$ is acyclic valued and $Q: X \rightarrow K(X)$ is a u.s.c. compact map i.e., $Q \in A C(X, X)$. Now from [3] 
there exists a $x_{0} \in X$ with $x_{0} \in Q\left(x_{0}\right)$. If $x_{0} \in X \backslash V$ then $x_{0} \in\left\{u_{0}\right\}$, a contradiction (note $u_{0} \in E$ and $E \subseteq V$ ). If $x_{0} \in \bar{V} \backslash E$ then since $Q: X \rightarrow K(E)$ (note $R: E \times[0,1] \rightarrow K(E)$ ) and $x_{0} \in Q\left(x_{0}\right)$ one has $x_{0} \in E$, a contradiction. Thus, $x_{0} \in E, r\left(x_{0}\right)=x_{0}, \lambda\left(x_{0}\right)=1$ so $x_{0} \in R\left(x_{0}, 1\right)=\Phi\left(x_{0}\right)$ i.e., (6) holds.

Remark 2. From the proof above, please note that one could replace $E$ is a (metrizable) ANR with any space provided the following hold: (i). E can be regarded as a closed subset of a normal space $X$, (ii). there exists an open neighborhood $V$ of $E$ in $X$ and a retraction $r: \bar{V} \rightarrow E$, and (iii). any map $\Psi \in A C(X, X)$ has a fixed point in X.

One can extend the above ideas to many other natural situations. In the remainder of this section, we will consider several extensions. Let $X$ be a (Hausdorff) topological vector space (so automatically Tychonoff), $Y$ a topological vector space, and $U$ an open subset of $X$. Also $L: \operatorname{dom} L \subseteq X \rightarrow Y$ is a linear (not necessarily continuous) single valued map where dom $L$ is a vector subspace of $X$ and finally let $T: X \rightarrow Y$ be a linear, continuous single valued map with $L+T: \operatorname{dom} L \rightarrow Y$ an isomorphism (i.e., a linear homeomorphism) and for convenience we say $T \in H_{L}(X, Y)$.

A map $F: \bar{U} \rightarrow 2^{Y}$ is said to be $(L, T)$ upper semicontinuous $\left((L, T)\right.$ u.s.c.) if $(L+T)^{-1}(F+T)$ : $\bar{U} \rightarrow K(X)$ is u.s.c. Now $F: \bar{U} \rightarrow 2^{Y}$ is said to be $(L, T)$ compact if $(L+T)^{-1}(F+T): \bar{U} \rightarrow 2^{X}$ is a compact map.

Definition 8. Write $F \in A C(\bar{U}, Y ; L, T)$ if $(L+T)^{-1}(F+T) \in A C(\bar{U}, X)$.

Definition 9. Write $F \in A C_{\partial U}(\bar{U}, Y ; L, T)$ if $F \in A C(\bar{U}, Y ; L, T)$ with $L x \notin F(x)$ for $x \in \partial U \cap$ dom $L$.

Definition 10. Consider two maps $F, G \in A C_{\partial U}(\bar{U}, Y ; L, T)$ and they are said to be homotopic in $A C_{\partial U}(\bar{U}, Y ; L, T)$, written $F \cong G$ in $A C_{\partial U}(\bar{U}, Y ; L, T)$, if there exists a $(L, T)$ u.s.c., $(L, T)$ compact mapping $N: \bar{U} \times[0,1] \rightarrow 2^{Y}$ such that $N_{t} \in A C_{\partial U}(\bar{U}, Y ; L, T)$ for each $t \in[0,1]$ and $N_{0}=F$ with $N_{1}=G\left(\right.$ where $N_{t}(u)=N(u, t)$.

Definition 11. A map $F \in A C_{\partial U}(\bar{U}, Y ; L, T)$ is L-essential in $A C_{\partial U}(\bar{U}, Y ; L, T)$ if for any $G \in$ $A C_{\partial U}(\bar{U}, Y ; L, T)$ with $\left.G\right|_{\partial U}=\left.F\right|_{\partial U}$ and with $F \cong G$ in $A C_{\partial U}(\bar{U}, Y ; L, T)$ there exists a $x \in U \cap \operatorname{dom} L$ with $L x \in G(x)$.

In $[2,4,5]$ we established the topological transversality theorem.

Theorem 6. Consider maps $F$ and $G$ in $A C_{\partial U}(\bar{U}, Y ; L, T)$ with $F \cong G$ in $A C_{\partial U}(\bar{U}, Y ; L, T)$. Now $F$ is L-essential in $A C_{\partial U}(\bar{U}, Y ; L, T)$ if and only if $G$ is L-essential in $A C_{\partial U}(\bar{U}, Y ; L, T)$.

We present the analogue of Theorem 2. Suppose (1) holds and $U$ is convex. Let $F, G$ be in $A C_{\partial U}(\bar{U}, Y ; L, T)$ with $\left.G\right|_{\partial U}=\left.F\right|_{\partial U}$. Then $F \cong G$ in $A C_{\partial U}(\bar{U}, Y ; L, T)$. To see this let $F^{\star}, \Lambda$ and $\Theta$ be as before Definition 5 Note $\Lambda$ and $\Theta$ are $(L, T)$ u.s.c. and $(L, T)$ compact mappings and $G \cong F^{\star}$ in $A C_{\partial U}(\bar{U}, Y ; L, T)$ (if $x \in \partial U \cap \operatorname{dom} L$ and $\lambda \in\left[0, \frac{1}{2}\right]$ with $L x \in \Lambda_{\lambda}(x)$ then $L x \in$ $G(2 \lambda x+(1-2 \lambda) x)=G(x)$, a contradiction) and $F^{\star} \cong F$ in $A C_{\partial U}(\bar{U}, Y ; L, T)$. Combining gives $F \cong G$ in $A C_{\partial U}(\bar{U}, Y ; L, T)$.

In this situation we replace Definition 11 with:

Definition 12. A map $F \in A C_{\partial U}(\bar{U}, Y ; L, T)$ is L-essential in $A C_{\partial U}(\bar{U}, Y ; L, T)$ if for any $G \in$ $A C_{\partial U}(\bar{U}, Y ; L, T)$ with $\left.G\right|_{\partial U}=\left.F\right|_{\partial U}$ there exists a $x \in U \cap \operatorname{dom} L$ with $L x \in G(x)$.

From Theorem 6 we have:

Theorem 7. Let $U$ be convex and suppose (1) holds. Consider two maps $F$ and $G$ in $A C_{\partial U}(\bar{U}, Y ; L, T)$ with $F \cong G$ in $A C_{\partial U}(\bar{U}, Y ; L, T)$. Now $F$ is L-essential (Definition 12) in $A C_{\partial U}(\bar{U}, Y ; L, T)$ if and only if $G$ is L-essential (Definition 12) in $A C_{\partial U}(\bar{U}, Y ; L, T)$.

Now we present the analogue of Theorem 4. 
Definition 13. Write $F \in A C(X, Y ; L, T)$ if $(L+T)^{-1}(F+T) \in A C(X, X)$.

Definition 14. Consider two maps $F, J \in A C(X, Y ; L, T)$ and they are said to be homotopic in $A C(X, Y ; L, T)$, written $F \cong J$ in $A C(X, Y ; L, T)$, if there exists a $(L, T)$ u.s.c., $(L, T)$ compact mapping $R: X \times[0,1] \rightarrow 2^{Y}$ with $R_{t} \in A C(X, Y ; L, T)$ for each $t \in[0,1], R_{1}=F$ and $R_{0}=J$ (where $R_{t}(x)=R(x, t)$ ).

Theorem 8. Let $X, Y, U, L$ and $T$ be as above and suppose $F \in A C_{\partial U}(\bar{U}, Y ; L, T)$. Assume $J \in$ $A C(X, Y ; L, T)$ and the following hold:

$$
L z \notin J(z) \text { for } z \in X \backslash(U \cap \operatorname{dom} L)
$$

$$
\text { there exists } y \in U \cap \operatorname{dom} L \text { with } L y \in J(y)
$$

$$
\begin{aligned}
& \text { for any } \Phi \in A C(X, Y ; L, T) \text { with } \Phi \cong J \text { in } A C(X, Y ; L, T) \\
& \text { there exists } z \in X \text { with } z \in(L+T)^{-1}(\Phi+T)(z) .
\end{aligned}
$$

Suppose $F \cong J$ in $A C_{\partial U}(\bar{U}, Y ; L, T)$. Then $F$ is L-essential (Definition 11) in $A C_{\partial U}(\bar{U}, Y ; L, T)$.

Proof. We show $J$ is L-essential (Definition 11) in $A C_{\partial U}(\bar{U}, Y ; L, T)$ (and then apply Theorem 6). Let $G \in A C_{\partial U}(\bar{U}, Y ; L, T)$ with $\left.G\right|_{\partial U}=\left.J\right|_{\partial U}$ and with $G \cong J$ in $A C_{\partial U}(\bar{U}, Y ; L, T)$. We need to show there exists a $x \in U \cap \operatorname{dom} L$ with $L x \in G(x)$. There exists (see Definition 10$)$ a $(L, T)$ u.s.c., $(L, T)$ compact mapping $\Lambda: \bar{U} \times[0,1] \rightarrow 2^{Y}$ with $\Lambda_{t} \in A C_{\partial U}(\bar{U}, Y ; L, T)$ for each $t \in[0,1], \Lambda_{0}=J$ and $\Lambda_{1}=G$ and let

$$
\begin{aligned}
\Omega & =\left\{x \in \bar{U} \cap \operatorname{dom} L: L x \in \Lambda_{t}(x) \text { for some } t \in[0,1]\right\} \\
& =\left\{x \in \bar{U}: x \in(L+T)^{-1}\left(\Lambda_{t}+T\right)(x) \text { for some } t \in[0,1]\right\} .
\end{aligned}
$$

Now $\Omega \neq \varnothing$ (see (8)) is compact, $\Omega \cap(X \backslash U)=\varnothing$, and since $X$ is Tychonoff there exists a (continuous) map $\sigma: X \rightarrow[0,1]$ with $\sigma(\Omega)=1$ and $\sigma(X \backslash U)=0$. Let $\Psi: X \times[0,1] \rightarrow 2^{Y}$ be

$$
\Psi(x, t)=\left\{\begin{array}{l}
\Lambda(x, t \sigma(x)), \quad x \in \bar{U} \\
J(x), \quad x \in X \backslash U .
\end{array}\right.
$$

Now $\Psi: X \times[0,1] \rightarrow 2^{Y}$ is a $(L, T)$ u.s.c., $(L, T)$ compact mapping and $\Psi_{t} \in A C(X, Y ; L, T)$ for each $t \in[0,1]$, so $\Psi_{1} \cong \Psi_{0}=J$ in $A C(X, Y ; L, T)$. Now from (9) there exists $x \in X$ with $x \in$ $(L+T)^{-1}\left(\Psi_{1}+T\right)(x)$. If $x \in X \backslash(U \cap \operatorname{dom} L)$ then $L x \in J(x)$ which contradicts (7). Consequently $x \in U \cap \operatorname{dom} L$ so $L x \in \Lambda(x, \sigma(x))$ and so $x \in \Omega, \sigma(x)=1$ and $L x \in \Lambda(x, 1)=G(x)$.

Next we consider a generalization of essential maps, namely the $d$-essential maps. Let $E$ be a completely regular topological space and $U$ an open subset of $E$.

Consider $F \in A C(\bar{U}, E)$ and write $F^{\star}=I \times F: \bar{U} \rightarrow K(\bar{U} \times E)$, here $I: \bar{U} \rightarrow \bar{U}$ is $I(x)=x$, and let

$$
d:\left\{\left(F^{\star}\right)^{-1}(B)\right\} \cup\{\varnothing\} \rightarrow D
$$

be any map with values in the nonempty set $D$ where $B=\{(x, x): x \in \bar{U}\}$.

Definition 15. Let $F \in A C_{\partial U}(\bar{U}, E)$ and write $F^{\star}=I \times F$. We write $F^{\star}: \bar{U} \rightarrow K(\bar{U} \times E)$ is d-essential if for any $J \in A C_{\partial U}(\bar{U}, E)$ (write $\left.J^{\star}=I \times J\right)$ and $\left.J\right|_{\partial U}=\left.F\right|_{\partial U}$ and $J \cong F$ in $A C_{\partial U}(\bar{U}, E$ ) we have $d\left(\left(F^{\star}\right)^{-1}(B)\right)=d\left(\left(J^{\star}\right)^{-1}(B)\right) \neq d(\varnothing)$. 
Remark 3. If $F^{\star}$ is d-essential then

$$
\varnothing \neq\left(F^{\star}\right)^{-1}(B)=\{x \in \bar{U}:(x, F x) \cap(x, x) \neq \varnothing\},
$$

so there exists a $x \in U$ with $(x, x) \in F^{\star}(x)$.

In [6] we established the topological transversality theorem.

Theorem 9. Consider two maps $\Phi$ and $\Psi$ in $A C_{\partial U}(\bar{U}, E)$ (write $\Phi^{\star}=I \times \Phi$ and $\left.\Psi^{\star}=I \times \Psi\right)$ with $\Phi \cong \Psi$ in $A C_{\partial U}(\bar{U}, E)$. Now $\Phi^{\star}$ is d-essential if and only if $\Psi^{\star}$ is d-essential.

We present the analogue of Theorem 2. Suppose $E$ is a (Hausdorff) topological vector space, $U$ is convex and assume $(1)$ holds. Let $F, G$ be in $A C_{\partial U}(\bar{U}, E)$ with $\left.G\right|_{\partial U}=\left.F\right|_{\partial U}$. Then before Definition 5 we showed $F \cong G$ in $A C_{\partial U}(\bar{U}, E)$. In this situation, we can replace Definition 15 with:

Definition 16. Let $F \in A C_{\partial U}(\bar{U}, E)$ and write $F^{\star}=I \times F$. We write $F^{\star}: \bar{U} \rightarrow K(\bar{U} \times E)$ is d-essential iffor any $J \in A C_{\partial U}(\bar{U}, E)$ (write $\left.J^{\star}=I \times J\right)$ and $\left.J\right|_{\partial U}=\left.F\right|_{\partial U}$ we have $d\left(\left(F^{\star}\right)^{-1}(B)\right)=d\left(\left(J^{\star}\right)^{-1}(B)\right) \neq$ $d(\varnothing)$.

From Theorem 9 we have:

Theorem 10. Let $E$ be a topological vector space, $U$ an open convex subset of $E$, and suppose (1) holds. Consider two maps $\Phi$ and $\Psi$ in $A C_{\partial U}(\bar{U}, E)$ (write $\Phi^{\star}=I \times \Phi$ and $\left.\Psi^{\star}=I \times \Psi\right)$ with $\Phi \cong \Psi$ in $A C_{\partial U}(\bar{U}, E)$. Now $\Phi^{\star}$ is d-essential (Definition 16) if and only if $\Psi^{\star}$ is d-essential (Definition 16).

Now we present the analogue of Theorem 4. and let

Consider $F \in A C(E, E)$ and write $F^{\star}=I \times F: E \rightarrow K(E \times E)$, here $I: E \rightarrow E$ is $I(x)=x$,

$$
d:\left\{\left(F^{\star}\right)^{-1}(\tilde{B})\right\} \cup\{\varnothing\} \rightarrow D
$$

be any map with values in the nonempty set $D$ where $\tilde{B}=\{(x, x): x \in E\}$.

Theorem 11. Let $E$ be a completely regular topological space, $U$ an open subset of $E, B=\{(x, x): x \in \bar{U}\}$, $\tilde{B}=\{(x, x): x \in E\}$ and $d$ is the map defined in (11). Suppose $F \in A C_{\partial U}(\bar{U}, E)$ (write $\left.F^{\star}=I \times F\right)$, $J \in A C(E, E)$ (write $\left.J^{\star}=I \times J\right)$ and (2) and (4) hold. Also suppose

$$
\begin{aligned}
& \text { for any } \left.\Phi \in A C(E, E) \text { (write } \Phi^{\star}=I \times \Phi\right) \text { with } \Phi \cong J \\
& \text { in } A C(E, E) \text { we have } d\left(\left(\Phi^{\star}\right)^{-1}(\tilde{B})\right)=d\left(\left(J^{\star}\right)^{-1}(\tilde{B})\right) \neq d(\varnothing)
\end{aligned}
$$

and $F \cong J$ in $A C_{\partial U}(\bar{U}, E)$. Then $F$ is d-essential (Definition 15).

Proof. We show $J$ is $d$-essential (Definition 15) (and then $F$ is $d$-essential (Definition 15) from Theorem 9). Let $G \in A C_{\partial U}(\bar{U}, E)$ (write $\left.G^{\star}=I \times G\right),\left.G\right|_{\partial U}=\left.J\right|_{\partial U}$ with $G \cong J$ in $A C_{\partial U}(\bar{U}, E)$. We need to show $d\left(\left(G^{\star}\right)^{-1}(B)\right)=d\left(\left(J^{\star}\right)^{-1}(B)\right) \neq d(\varnothing)$. There exists (see Definition 3) a u.s.c. compact map $\Lambda: \bar{U} \times[0,1] \rightarrow K(E)$ with $\Lambda_{t} \in A C_{\partial U}(\bar{U}, E)$ for each $t \in[0,1], \Lambda_{0}=J$ and $\Lambda_{1}=G$. Let $\Lambda^{\star}: \bar{U} \times[0,1] \rightarrow K(\bar{U} \times E)$ be $\Lambda^{\star}(x, t)=(x, \Lambda(x, t))$ and let

$$
\Omega=\left\{x \in \bar{U}:(x, x) \in \Lambda_{t}^{\star}(x) \text { for some } t \in[0,1]\right\} .
$$

Notice $\Omega \neq \varnothing$ (see (4)) is compact and $\Omega \cap(E \backslash U)=\varnothing$. Thus, there exists a continuous function $\sigma: E \rightarrow[0,1]$ with $\sigma(\Omega)=1$ and $\sigma(E \backslash U)=0$. Let $\Psi: E \times[0,1] \rightarrow K(E)$ be

$$
\Psi(x, t)=\left\{\begin{array}{l}
\Lambda(x, t \sigma(x)), \quad x \in \bar{U} \\
J(x), \quad x \in E \backslash U .
\end{array}\right.
$$


Note $\Psi: E \times[0,1] \rightarrow K(E)$ is a u.s.c. compact map with $\Psi_{t} \in A C(E, E)$ for each $t \in[0,1]$, so $\Psi_{1} \cong \Psi_{0}=J$ in $A C(E, E)$. Write $\Psi_{1}^{\star}=I \times \Psi_{1}$ and (12) implies

$$
d\left(\left(\Psi_{1}^{\star}\right)^{-1}(\tilde{B})\right)=d\left(\left(J^{\star}\right)^{-1}(\tilde{B})\right) \neq d(\varnothing) .
$$

Note from (2) that

$$
\begin{aligned}
\left(J^{\star}\right)^{-1}(\tilde{B}) & =\{x \in E:(x, x) \cap(x, J(x)) \neq \varnothing\}=\{x \in \bar{U}:(x, x) \cap(x, J(x)) \neq \varnothing\} \\
& =\left(J^{\star}\right)^{-1}(B)
\end{aligned}
$$

and also from (2) (note $\Psi_{1}(x)=J(x)$ for $\left.x \in E \backslash U\right)$ that

$$
\begin{aligned}
\left(\Psi_{1}^{\star}\right)^{-1}(\tilde{B}) & =\left\{x \in E:(x, x) \cap\left(x, \Psi_{1}(x)\right) \neq \varnothing\right\}=\left\{x \in \bar{U}:(x, x) \cap\left(x, \Psi_{1}(x)\right) \neq \varnothing\right\} \\
& =\left(\Psi_{1}^{\star}\right)^{-1}(B)
\end{aligned}
$$

SO

$$
d\left(\left(\Psi_{1}^{\star}\right)^{-1}(B)\right)=d\left(\left(J^{\star}\right)^{-1}(B)\right) \neq d(\varnothing) .
$$

Finally, note $\sigma(\Omega)=1\left(\right.$ note $\left.(x, \Lambda(x, \sigma(x)))=\Lambda_{\sigma(x)}^{\star}(x)\right)$ so

$$
\begin{aligned}
\left(\Psi_{1}^{\star}\right)^{-1}(\tilde{B}) & =\{x \in \bar{U}:(x, x) \cap(x, \Lambda(x, \sigma(x))) \neq \varnothing\}=\{x \in \bar{U}:(x, x) \cap(x, \Lambda(x, 1)) \neq \varnothing\} \\
& =\left(G^{\star}\right)^{-1}(B)
\end{aligned}
$$

and as a result

$$
d\left(\left(G^{\star}\right)^{-1}(B)\right)=d\left(\left(J^{\star}\right)^{-1}(B)\right) \neq d(\varnothing) .
$$

Next we consider a generalization of $L$-essential maps, namely the $d$ - $L$-essential maps. Let $X, Y, U, L$ and $T$ be as described after Remark 2 .

Consider $F \in A C(\bar{U}, Y ; L, T)$ and write $F^{\star}=I \times(L+T)^{-1}(F+T): \bar{U} \rightarrow K(\bar{U} \times X)$, here $I: \bar{U} \rightarrow \bar{U}$ is $I(x)=x$, and let

$$
d:\left\{\left(F^{\star}\right)^{-1}(B)\right\} \cup\{\varnothing\} \rightarrow D
$$

be any map with values in the nonempty set $D$ where $B=\{(x, x): x \in \bar{U}\}$.

Definition 17. Let $F \in A C_{\partial U}(\bar{U}, Y ; L, T)$ and write $F^{\star}=I \times(L+T)^{-1}(F+T)$. We write $F^{\star}: \bar{U} \rightarrow$ $K(\bar{U} \times X)$ is d-L-essential if for any $J \in A C_{\partial U}(\bar{U}, Y ; L, T)$ (write $J^{\star}=I \times(L+T)^{-1}(J+T)$ ) with $\left.J\right|_{\partial U}=\left.F\right|_{\partial U}$ and $J \cong F$ in $A C_{\partial U}(\bar{U}, Y ; L, T)$ we have $d\left(\left(F^{\star}\right)^{-1}(B)\right)=d\left(\left(J^{\star}\right)^{-1}(B)\right) \neq d(\varnothing)$.

Remark 4. If $F^{\star}$ is $d$-L-essential then

$$
\varnothing \neq\left(F^{\star}\right)^{-1}(B)=\left\{x \in \bar{U}:\left(x,(L+T)^{-1}(F+T)(x)\right) \cap(x, x) \neq \varnothing\right\},
$$

so there exists a $x \in U \cap \operatorname{dom} L$ with $(x, x) \in F^{\star}(x)$.

In [5] we established the topological transversality theorem.

Theorem 12. Consider maps $\Phi$ and $\Psi$ in $A C_{\partial U}(\bar{U}, Y ; L, T)$ (write $\Phi^{\star}=I \times(L+T)^{-1}(\Phi+T)$ and $\left.\Psi^{\star}=I \times(L+T)^{-1}(\Psi+T)\right)$ with $\Phi \cong \Psi$ in $A C_{\partial U}(\bar{U}, Y ; L, T)$. Now $\Phi^{\star}$ is d-L-essential if and only if $\Psi^{\star}$ is $d$-L-essential. 
We present the analogue of Theorem 2. Suppose (1) holds and $U$ is convex. Let $F, G$ be in $A C_{\partial U}(\bar{U}, Y ; L, T)$ with $\left.G\right|_{\partial U}=\left.F\right|_{\partial U}$. Then after Theorem 6 we showed $F \cong G$ in $A C_{\partial U}(\bar{U}, Y ; L, T)$. In this situation we can replace Definition 17 with:

Definition 18. Let $F \in A C_{\partial U}(\bar{U}, Y ; L, T)$ and write $F^{\star}=I \times(L+T)^{-1}(F+T)$. We write $F^{\star}: \bar{U} \rightarrow$ $K(\bar{U} \times X)$ is $d$-L-essential if for any $J \in A C_{\partial U}(\bar{U}, Y ; L, T)$ (write $J^{\star}=I \times(L+T)^{-1}(J+T)$ ) with $\left.J\right|_{\partial U}=\left.F\right|_{\partial U}$ we have $d\left(\left(F^{\star}\right)^{-1}(B)\right)=d\left(\left(J^{\star}\right)^{-1}(B)\right) \neq d(\varnothing)$.

From Theorem 12 we have:

Theorem 17. Let $U$ be convex and suppose (1) holds. Consider two maps $\Phi$ and $\Psi$ in $A C_{\partial U}(\bar{U}, Y ; L, T)$ (write $\Phi^{\star}=I \times(L+T)^{-1}(\Phi+T)$ and $\Psi^{\star}=I \times(L+T)^{-1}(\Psi+T)$ ) with $\Phi \cong \Psi$ in $A C_{\partial U}(\bar{U}, Y ; L, T)$. Now $\Phi^{\star}$ is d-L-essential (Definition 18) if and only if $\Psi^{\star}$ is $d$-L-essential (Definition 18).

Finally, we present the analogue of Theorem 4. Consider $F \in A C(X, Y ; L, T)$ and write $F^{\star}=$ $I \times(L+T)^{-1}(F+T): X \rightarrow K(X \times X)$, here $I: X \rightarrow X$ given is $I(x)=x$, and let

$$
d:\left\{\left(F^{\star}\right)^{-1}(\tilde{B})\right\} \cup\{\varnothing\} \rightarrow D
$$

be any map with values in the nonempty set $D$ where $\tilde{B}=\{(x, x): x \in X\}$.

Theorem 18. Let $X, Y, U, L$ and $T$ be as above, $B=\{(x, x): x \in \bar{U}\}, \tilde{B}=\{(x, x): x \in X\}$ and $d$ is the map defined in (14). Suppose $F \in A C_{\partial U}(\bar{U}, Y ; L, T)$ (write $F^{\star}=I \times(L+T)^{-1}(F+T)$ ), $J \in A C(X, Y ; L, T)$ (write $J^{\star}=I \times(L+T)^{-1}(J+T)$ ) and $(7)$ and $(8)$ hold. In addition assume

$$
\begin{aligned}
& \text { for any } \Phi \in A C(X, Y ; L, T) \text { (write } \Phi^{\star}=I \times(L+T)^{-1}(\Phi+T) \text { ) } \\
& \text { with } \Phi \cong J \text { in } A C(X, Y ; L, T) \text { we have } \\
& d\left(\left(\Phi^{\star}\right)^{-1}(\tilde{B})\right)=d\left(\left(J^{\star}\right)^{-1}(\tilde{B})\right) \neq d(\varnothing),
\end{aligned}
$$

and $F \cong J$ in $A C_{\partial U}(\bar{U}, Y ; L, T)$. Then $F$ is d-L-essential (Definition 17).

Proof. We show $J$ is $d$ - $L$-essential (Definition 17) (and then apply Theorem 12). Let $G \in$ $A C_{\partial U}(\bar{U}, Y ; L, T)$ and write $G^{\star}=I \times(L+T)^{-1}(G+T),\left.G\right|_{\partial U}=\left.J\right|_{\partial U}$ with $G \cong J$ in $A C_{\partial U}(\bar{U}, Y ; L, T)$. Now there exists (Definition 10) a $(L, T)$ u.s.c., $(L, T)$ compact map $\Lambda: \bar{U} \times[0,1] \rightarrow$ $2^{Y}$ with $\Lambda_{t} \in A C_{\partial U}(\bar{U}, Y ; L, T)$ for each $t \in[0,1], \Lambda_{0}=J$ and $\Lambda_{1}=G$. Let $\Lambda^{\star}: \bar{U} \times[0,1] \rightarrow$ $K(\bar{U} \times X)$ be $\Lambda^{\star}(x, t)=\left(x,(L+T)^{-1}\left(\Lambda_{t}+T\right)(x)\right)$ and let

$$
\Omega=\left\{x \in \bar{U}:(x, x) \in \Lambda_{t}^{\star}(x) \text { for some } t \in[0,1]\right\} .
$$

Note $\Omega \neq \varnothing$ (see (8)) is compact, $\Omega \cap(X \backslash U)=\varnothing$ and since $X$ is Tychonoff there exists a (continuous) $\operatorname{map} \sigma: X \rightarrow[0,1]$ with $\sigma(\Omega)=1$ and $\sigma(X \backslash U)=0$. Let $\Psi: X \times[0,1] \rightarrow 2^{Y}$ by

$$
\Psi(x, t)=\left\{\begin{array}{l}
\Lambda(x, t \sigma(x)), \quad x \in \bar{U} \\
J(x), \quad x \in X \backslash U
\end{array}\right.
$$

Note $\Psi: X \times[0,1] \rightarrow 2^{Y}$ is a $(L, T)$ u.s.c., $(L, T)$ compact map with $\Psi_{t} \in A C(X, Y ; L, T)$ for each $t \in[0,1]$, so $\Psi_{1} \cong \Psi_{0}=J$ in $A C(X, Y ; L, T)$. Write $\Psi_{1}^{\star}=I \times(L+T)^{-1}\left(\Psi_{1}+T\right)$ and (15) implies

$$
d\left(\left(\Psi_{1}^{\star}\right)^{-1}(\tilde{B})\right)=d\left(\left(J^{\star}\right)^{-1}(\tilde{B})\right) \neq d(\varnothing) .
$$


Note from (7) that

$$
\begin{aligned}
\left(J^{\star}\right)^{-1}(\tilde{B}) & =\left\{x \in X:(x, x) \cap\left(x,(L+T)^{-1}(J+T)(x)\right) \neq \varnothing\right\} \\
& =\left\{x \in \bar{U}:(x, x) \cap\left(x,(L+T)^{-1}(J+T)(x)\right) \neq \varnothing\right\} \\
& =\left(J^{\star}\right)^{-1}(B)
\end{aligned}
$$

and also from (7) (note $\Psi_{1}(x)=J(x)$ for $\left.x \in X \backslash U\right)$ we have

$$
\begin{aligned}
\left(\Psi_{1}^{\star}\right)^{-1}(\tilde{B}) & =\left\{x \in X:(x, x) \cap\left(x,(L+T)^{-1}\left(\Psi_{1}+T\right)(x)\right) \neq \varnothing\right\} \\
& =\left\{x \in \bar{U}:(x, x) \cap\left(x,(L+T)^{-1}\left(\Psi_{1}+T\right)(x)\right) \neq \varnothing\right\} \\
& =\left(\Psi_{1}^{\star}\right)^{-1}(B)
\end{aligned}
$$

so

$$
d\left(\left(\Psi_{1}^{\star}\right)^{-1}(B)\right)=d\left(\left(J^{\star}\right)^{-1}(B)\right) \neq d(\varnothing) .
$$

Finally, note $\sigma(\Omega)=1\left(\right.$ note $\left.\left.\left(x,(L+T)^{-1}\left(\Lambda_{\sigma(x)}+T\right)(x)\right)\right)=\Lambda_{\sigma(x)}^{\star}(x)\right)$ so

$$
\begin{aligned}
\left(\Psi_{1}^{\star}\right)^{-1}(\tilde{B}) & \left.=\left\{x \in \bar{U}:(x, x) \cap\left(x,(L+T)^{-1}\left(\Lambda_{\sigma(x)}+T\right)(x)\right)\right) \neq \varnothing\right\} \\
& \left.=\left\{x \in \bar{U}:(x, x) \cap\left(x,(L+T)^{-1}\left(\Lambda_{1}+T\right)(x)\right)\right) \neq \varnothing\right\} \\
& =\left(G^{\star}\right)^{-1}(B)
\end{aligned}
$$

and so

$$
d\left(\left(G^{\star}\right)^{-1}(B)\right)=d\left(\left(J^{\star}\right)^{-1}(B)\right) \neq d(\varnothing)
$$

Funding: This research received no external funding.

Conflicts of Interest: The author declares no conflict of interest.

\section{References}

1. Granas, A. Sur la méthode de continuité de Poincare. C. R. Acad. Sci. Paris 1976, 282, 983-985.

2. O'Regan, D. Continuation principles based on essential maps and topological degree. Appl. Anal. 2015, 94, 1032-1041. [CrossRef]

3. Precup, R. On the topological transversality principle. Nonlinear Anal. 1993, 20, 1-9. [CrossRef]

4. O'Regan, D. Continuation theorems for acyclic maps in topological spaces. Commun. Appl. Anal. 2009, 13, 39-45.

5. O'Regan, D. A unified theory for homotopy principles for multimaps. Appl. Anal. 2013, 92, 1944-1958. [CrossRef]

6. O'Regan, D. Homotopy principles for $d$-essential maps. J. Nonlinear Convex Anal. 2013, 14, 415-422.

7. Agarwal, R.P.; O'Regan, D. A note on the topological transversality theorem for acyclic maps. Appl. Math. Lett. 2005, 18, 17-22. [CrossRef]

8. He, J.H.; Ji, F.Y. Taylor series solution for Lane-Emden equation. J. Math. Chem. 2019. [CrossRef]

9. O'Regan, D.; Precup, R. Theorems of Leray-Schauder Type and Applications; Gordon and Breach Publishers: Amsterdam, The Netherlands, 2001.

10. Gorniewicz, L. Topological Fixed Point Theory of Multivalued Mappings; Kluwer Acad. Publishers: Dordrecht, The Netherlands, 1999. 
11. O'Regan, D. Fixed point theory on extension type spaces and essential maps on topological spaces. Fixed Point Theory Appl. 2004, 1, 13-20. [CrossRef]

12. Aliprantis, C.D.; Border, K.C. Infinite-Dimensional Analysis. Studies in Economic Theory; Springer: Berlin, Germany, 1994; Volume 4. 